\title{
Pesca exploratoria con trampas alrededor de las islas Robinson Crusoe y Santa Clara, archipiélago de Juan Fernández, Chile*
}

\author{
Patricio M. Arana \\ Escuela de Ciencias del Mar, Universidad Católica de Valparaíso \\ Casilla 1020, Valparaíso, Chile \\ E-mail: parana@ucv.cl
}

Recibido: 4 marzo 1999; versión corregida: 12 agosto 1999; aceptado: 13 septiembre 1999

\begin{abstract}
RESUMEN. Con el objeto de buscar recursos alternativos a la langosta de Juan Fernández (Jasus frontalis), que constituye el objetivo tradicional de los pescadores artesanales, se efectuó una campaña de pesca exploratoria con trampas en torno a las islas Robinson Crusoe y Santa Clara (Archipiélago de Juan Fernández, Chile). Entre el 11 de noviembre de 1996 y el 23 de marzo de 1997 se realizaron un total de 71 salidas de pesca, en las que se caló un total de 241 trampas, entre 50 y $1000 \mathrm{~m}$ de profundidad. En estas experiencias se utilizaron distintos diseños de trampas: troncocónico, tetragonal y piramidal, de diferente tamaño y ubicación de las entradas, y trampas langosteras con entrada en un extremo y en la parte superior. Las dos últimas, las utilizan los pescadores del archipiélago para la extracción de la langosta. Las trampas troncocónicas, tetragonales y piramidales fueron caladas con entradas circulares o rectangulares.

Los únicos crustáceos capturados correspondieron a: langosta de Juan Fernández (Jasus frontalis), centolla de Juan Fernández (Paromola rathbuni), jaiba remadora (Ovalipes trimaculatus) y cangrejo dorado (Chaceon chilensis). La langosta se capturó entre 50 y 100 m; la centolla de Juan Fernández entre 100 y 300 m; el cangrejo dorado entre 200 y $1000 \mathrm{~m}$; mientras que la jaiba remadora se encontró sólo en el veril de $100 \mathrm{~m}$. El único recurso que presenta interés pesquero es $C$. chilensis, tanto por su abundancia como por la amplia distribución en profundidad y en torno a las islas analizadas.
\end{abstract}

Palabras claves: pesca exploratoria, trampas, crustáceos, archipiélago de Juan Fernández, Chile.

\section{Exploratory survey with pots around Robinson Crusoe and Santa Clara islands, Juan Fernandez archipelago, Chile*}

\begin{abstract}
Looking for alternative resources to the Juan Fernandez spiny rock lobster (Jasus frontalis), the traditional target species of the artisanal fishermen of the archipelago, an exploratory fishing campaign with fishing pots was conducted around Robinson Crusoe and Santa Clara islands. Between November 11, 1996, and March 23, 1997, a total of 71 fishing journeys were conducted, during which 241 pots were set between 50 and $1000 \mathrm{~m}$ depth. In these experiences different pot designs were used: conical-trunk, tetragonal, piramidal, and lobster pots with a wicket on one extreme and lobster pot with an upper wicket. The last two, are the ones often used by the archipelago fishermen to catch lobsters. The conical-trunk, tetragonal and piramidal pots were with circular or rectangular wickets.

The resources caught were: Juan Fernandez spiny rock lobster (Jasus frontalis), Juan Fernandez king crab (Paromola rathbuni), rowing crab (Ovalipes trimaculatus) and golden crab (Chaceon chilensis). The spiny rock lobster was captured between 50 and $100 \mathrm{~m}$ depth, while the rowing crab was found only in the $100 \mathrm{~m}$ isobath; Juan Fernandez king crab between 100 and $300 \mathrm{~m}$ and the golden crab between 200 and $1000 \mathrm{~m}$. The only resource with fishing interest is $C$. chilensis, both by its abundance as well as for its wide distribution in depth and around these islands.
\end{abstract}

Key words: exploratory fishing, pots, crustaceans, Juan Fernandez archipelago, Chile.

* Artículo generado como parte del "Programa de pesca exploratoria y experimental de recursos pesqueros alternativos a la langosta en las islas Robinson Crusoe y Santa Clara” (Proyecto FIP 95/25), financiado por el Fondo de Investigación Pesquera y realizado por la Universidad Católica de Valparaíso. 


\section{INTRODUCCIÓN}

El archipiélago de Juan Fernández (3340'S$78^{\circ} 50^{\prime} \mathrm{W}$ ), está constituido por las islas Robinson Crusoe, Santa Clara y Alejandro Selkirk, esta última a 90 millas náuticas al oeste de las dos primeras. La única población permanente se encuentra en la isla Robinson Crusoe, con alrededor de 600 habitantes. La economía de este archipiélago se caracteriza por ser simple y monoproductiva, sustentada casi exclusivamente por la captura y comercialización de la "langosta de Juan Fernández" (Jasus frontalis $\mathrm{H}$. Milne Edwards, 1837). Dicha actividad es ejercida por aproximadamente 150 pescadores artesanales, percibiendo rentas que proporcionan el sustento económico a gran parte de la población. La captura de langosta es realizada con trampas rectangulares de dos cámaras, construidas manualmente con maderas locales, utilizando embarcaciones artesanales, la mayoría equipados con motores fuera de borda y algunos con motores alojados en su interior.

El alto nivel de dependencia de la economía de estas islas, respecto a la pesquería de este crustáceo reviste permanente preocupación, ya que ésta queda sometida a las fluctuaciones en la abundancia que presenta anualmente dicho recurso, el que ha presentado en los últimos años niveles de captura notoriamente reducidos. Hasta ahora, las especies comerciales alternativas a la langosta en el archipiélago son escasas y con volúmenes de captura que no permiten mantener por sí solo una fuente laboral estable. Cabe destacar, además, que los pescadores que operan en estas islas se caracterizan por trabajar bajo la condición de pequeños empresarios independientes, propietarios de diversos bienes de capital que les permiten realizar las faenas de pesca durante cada temporada.

Otro aspecto que se debe tener en consideración, es el hecho de que todas las faenas pesqueras que se realizan en este archipiélago son de carácter manual, sin utilización de mecanización, salvo los motores de propulsión de las embarcaciones. A lo menos en parte, esto explica porqué hasta ahora no se efectúen faenas extractivas a mayores profundidades, no obstante que se conoce la existencia de al menos la centolla de Juan Fernández (Paromola rathbuni Porter, 1908) y del cangrejo dorado (Chaceon chilensis Chirino-Gálvez y Manning, 1989), crustáceos que se extraen ocasionalmente en estas islas (Arana, 1987), aunque se desconoce el rango de distribución en profundidad y la abundancia de estas especies.
De acuerdo a lo señalado precedentemente, se destaca la importancia de encontrar recursos que permitan diversificar la actual pesquería centrada en la extracción de la langosta. Al respecto, el presente trabajo tuvo como principales objetivos el investigar la presencia y distribución de crustáceos en los alrededores de las islas Robinson Crusoe y Santa Clara y establecer si éstos son susceptibles de ser explotados por los pescadores de estas islas.

\section{MATERIALES Y MÉTODOS}

Con el fin de explorar la plataforma y el talud continental común de las islas Robinson Crusoe y Santa Clara (Archipiélago de Juan Fernández), se establecieron estaciones de muestreo en diferentes caras de estas islas, eligiéndose aquellos lugares en que se encontraba el rango de profundidad a estudiar (50 a $1000 \mathrm{~m}$ ) a menor distancia de la costa. De este modo, se definieron cuatro transectas, ubicadas respectivamente frente a Puerto Francés (T1), Islote Juanango (T2), Bahía El Padre (T3) e islote El Verdugo (T4). Dado que la investigación se orientó a la búsqueda de crustáceos bentónicos, se utilizaron diversos tipos de trampas las que se calaron en estaciones que se posicionaron sobre los veriles de 50, $100,200,300,400,500,600,800$ y $1000 \mathrm{~m}$ de profundidad (Fig.1).

Las faenas de pesca se iniciaron en la lancha "Natalia", y posteriormente se continuaron con los botes "Independencia", "Clara Margarita", "Norma" y "Kibalión", que corresponden a embarcaciones artesanales semejantes a botes balleneros de doble proa de 8 a $10 \mathrm{~m}$ de eslora, equipadas con motores fuera de borda dispuestos en un sistema de cajón interno. Cada embarcación fue equipada con un virador hidráulico de doble plato accionado por un motor bencinero de 6,5 HP, un posicionador satelital GPS y un ecosonda con alcance superior a $1000 \mathrm{~m}$.

El calado de las trampas se efectuó en forma individual y formando tenas, lo que dependió de factores operativos al momento de realizar el calado, tales como la intensidad de las corrientes, profundidad de trabajo, capturas obtenidas y capacidad de virado del winche hidráulico. El número de trampas caladas en cada día de trabajo (estrato de profundidad), dependió del tiempo asociado a la maniobra de virado y de las condiciones de operación mencionadas anteriormente. En cada estación se realizaron varias caladas con cada tipo de trampa, tratando de completar un cierto número de réplicas 


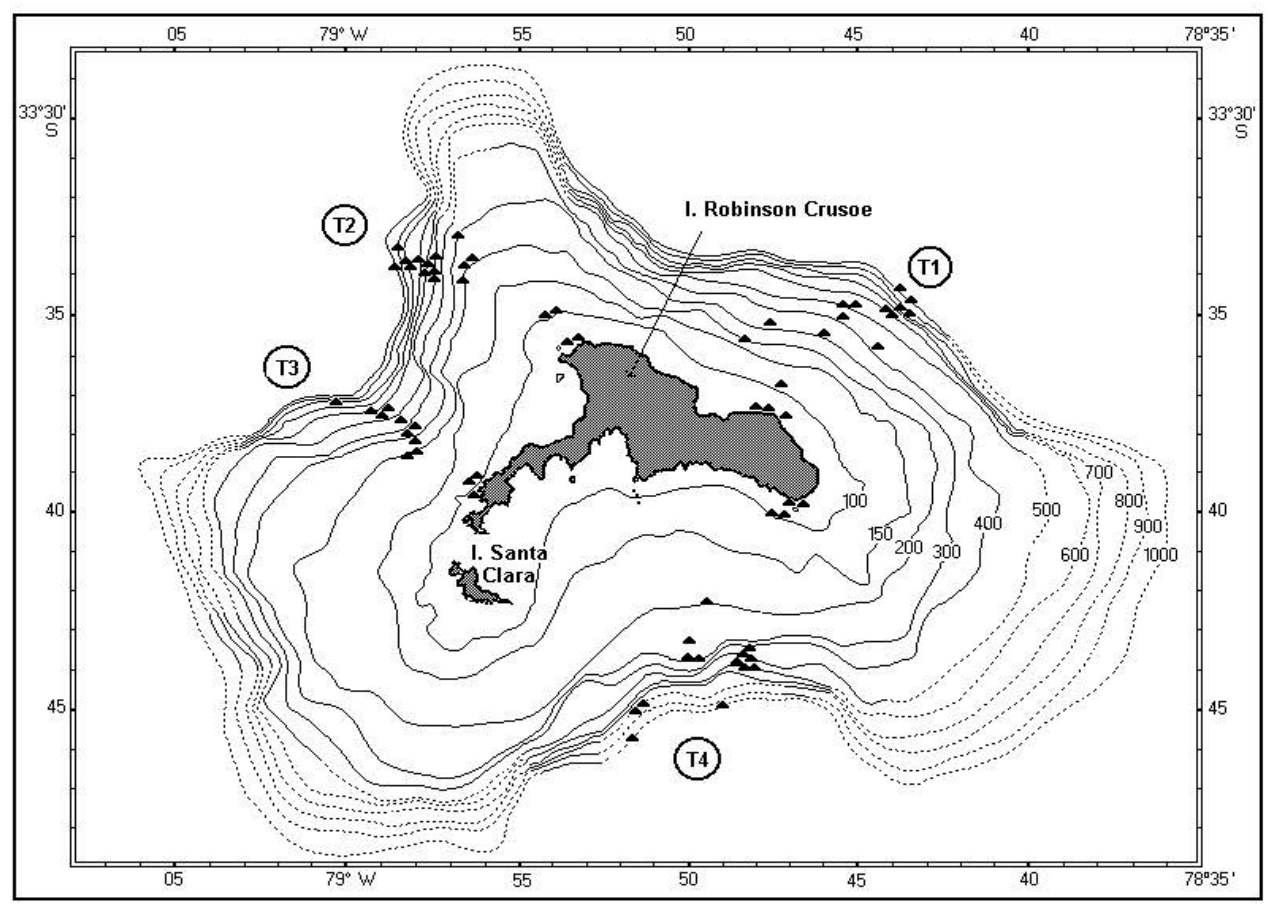

Figura 1. Transectas (T) y estaciones ( $\Delta$ ) donde se calaron trampas durante la pesca exploratoria.

Figure 1. Transects $(T)$ and stations $(\Delta)$ where pots were set during the fishing survey.

T1: Puerto Francés, T2: Islote Juanango, T3: Bahía El Padre, T4: Islote El Verdugo

en cada una de ellas (>6). Se programó la revisión de los aparejos cada 24 horas, con el objeto de retirar las capturas, renovar la carnada y volverlos a calar ya sea en el mismo lugar o en la siguiente estación de muestreo.

En atención a que se utilizaron embarcaciones de pequeño tamaño, se trabajaría a gran profundidad, desconociéndose las especies que se podrían capturar, se diseñaron y construyeron diferentes tipos de trampas para ser probadas, a fin de seleccionar aquellas que por su diseño y operatividad se adecuaran a las condiciones de trabajo que se encontraran en terreno. Los diseños de trampa utilizados fueron los siguientes (Figs. 2 y 3 ):

1. Troncocónica con entrada rectangular (A1): Construida de $60 \mathrm{~cm}$ de alto, $100 \mathrm{~cm}$ de diámetro en su base inferior y $50 \mathrm{~cm}$ en su base superior, con barras de fierro dulce de sección circular de $10,5 \mathrm{~mm}$ de diámetro, soldadas al arco y cubierta con paños de red de poliamida torcida $24 / 210$ de longitud de malla de 3,81 cm (1,5 pulgadas). La entrada o boca, ubicada en la parte superior de la trampa, fue con- feccionada en forma rectangular de $30 \times 10 \mathrm{~cm}$, utilizando varillas de fierro de $0,6 \mathrm{~mm}$ de sección.

2. Troncocónica con entrada circular (A2): De las mismas dimensiones y características que el diseño anterior, la entrada o boca, ubicada en la parte superior de la trampa, fue confeccionada en forma de anillo de $25 \mathrm{~cm}$ de diámetro, utilizando varillas de fierro de $0,6 \mathrm{~mm}$ de sección.

3. Tetragonal pequeña con entrada rectangular (B1): Con $48 \mathrm{~cm}$ de alto, $56 \mathrm{~cm}$ de ancho y $80 \mathrm{~cm}$ de largo, este modelo fue construido con barras de fierro dulce de sección cuadrada de $14 \mathrm{~mm}$ de espesor, soldadas al arco y cubierta con paños de red de poliamida torcida $24 / 210$ de longitud de malla de $3,81 \mathrm{~cm}$ (1,5 pulgadas). La entrada o boca, ubicada en la pared lateral de la trampa a aproximadamente $20 \mathrm{~cm}$ del piso, fue confeccionada en forma rectangular de 30x10 cm, utilizando varillas de fierro de $0,6 \mathrm{~mm}$ de sección.

4. Tetragonal pequeña con entrada circular (B2): Manteniendo las mismas dimensiones y caracterís- 


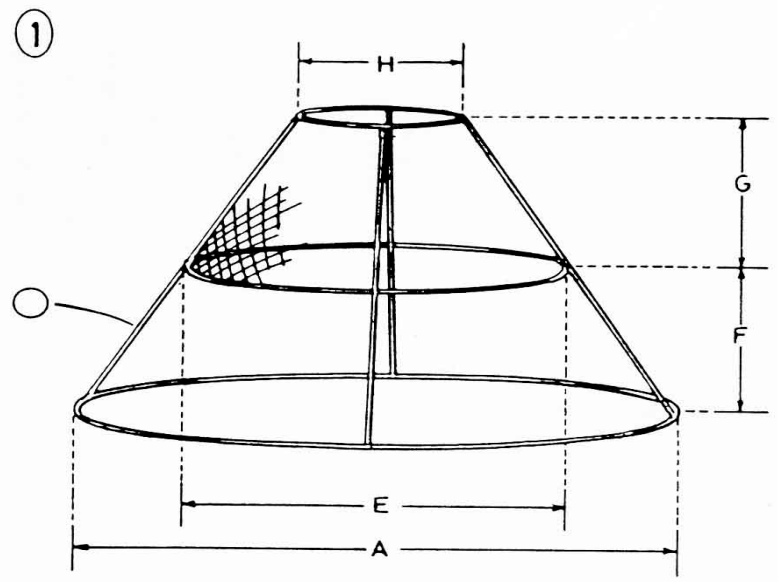

Dimensiones geometricas y materiales
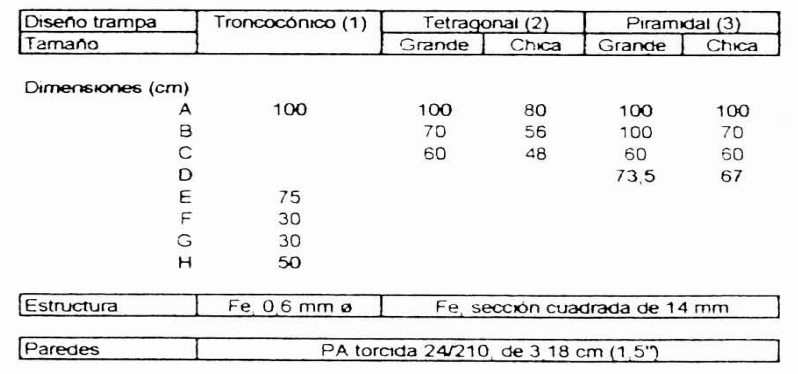

(2)

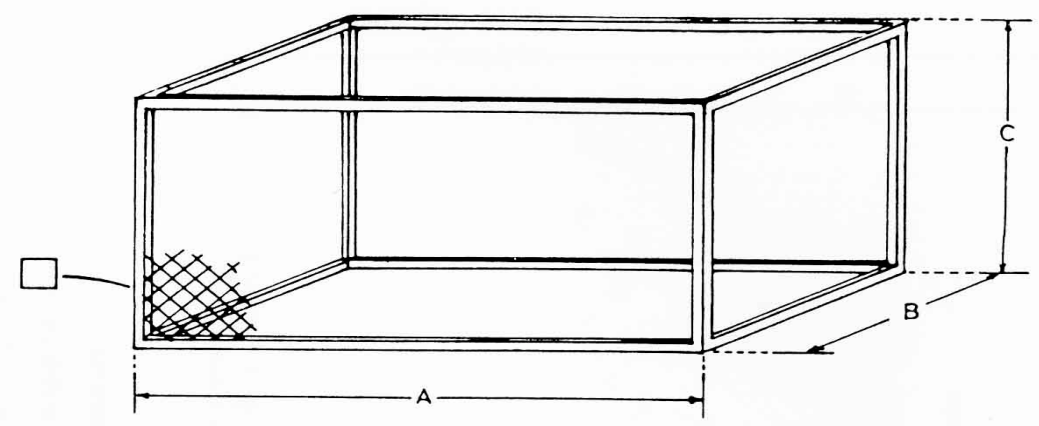

(3)

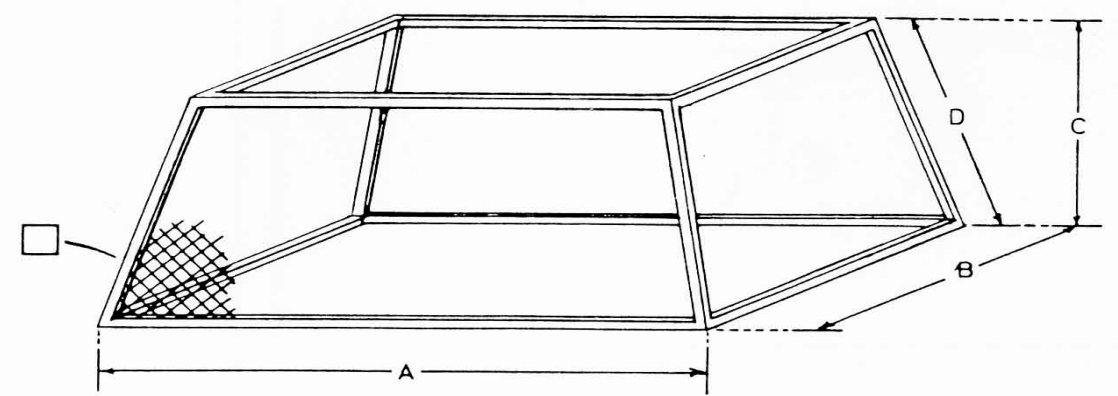

Figura 2. Diseños de trampas utilizadas en la pesca exploratoria y experimental (troncocónico, tetragonal y piramidal).

Figure 2. Pot designs used in the exploratory and experimental fishing (conical-trunk, tetragonal and piramidal pots). 

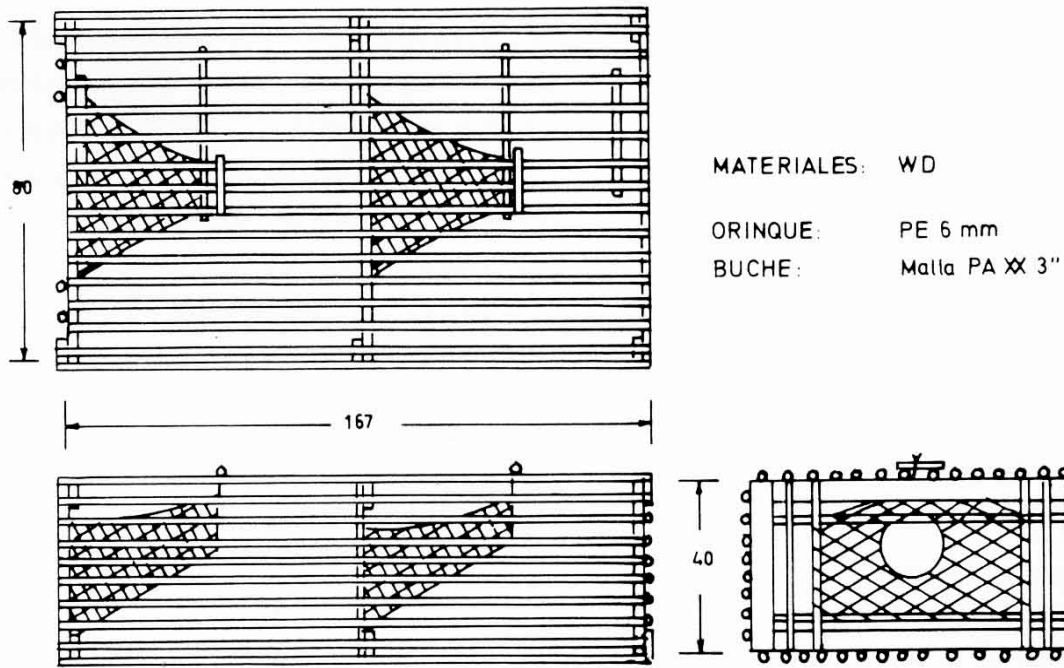

Trampa langostera con entrada en un extremo

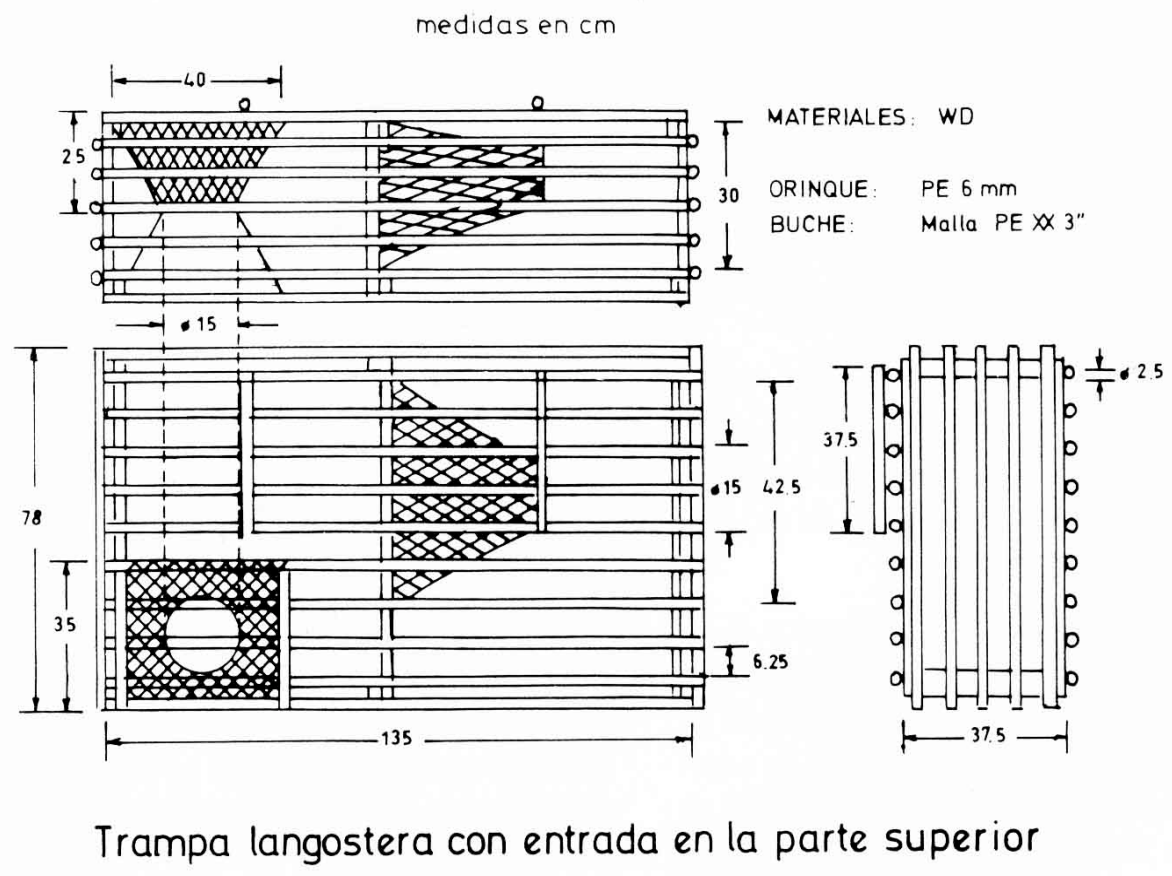

Figura 3. Diseños de trampas langosteras utilizadas en la pesca exploratoria y experimental. Figure 3. Lobster pot designs used in the exploratory and experimental fishing. 
ticas que el modelo B1, sólo difiere en la entrada o boca, la cual está ubicada en la pared lateral de la trampa a aproximadamente $20 \mathrm{~cm}$ del piso y confeccionada en forma de anillo de $25 \mathrm{~cm}$ de diámetro, utilizando varillas de fierro de $0,6 \mathrm{~mm}$ de sección.

5. Tetragonal grande con entrada rectangular $(\mathrm{C} 1)$ : $(60 \times 70 \times 100 \mathrm{~cm})$, construida con barras de fierro dulce de sección cuadrada de $14 \mathrm{~mm}$ de espesor, soldadas al arco y cubierta con paños de red de poliamida torcida $24 / 210$ de longitud de malla de $3,81 \mathrm{~cm}$ (1,5 pulgadas). La entrada o boca, ubicada en la pared lateral de la trampa a aproximadamente $20 \mathrm{~cm}$ del piso, fue confeccionada en forma rectangular de 30x10 cm, utilizando varillas de fierro de 0,6 mm de sección.

6. Tetragonal grande con entrada circular (C2): Similar al diseño $\mathrm{C} 1$, sólo que la entrada o boca, ubicada en la pared lateral de la trampa a aproximadamente $20 \mathrm{~cm}$ del piso, fue confeccionada en forma de anillo de $25 \mathrm{~cm}$ de diámetro, utilizando varillas de fierro de 0,6 $\mathrm{mm}$ de sección.

7. Piramidal pequeña con entrada rectangular (D1): De $100 \times 70 \mathrm{~cm}$ de base inferior, $60 \mathrm{~cm}$ de alto y $60 \times 30 \mathrm{~cm}$ de base superior, este modelo fue confeccionado con barras de fierro dulce de sección cuadrada de $14 \mathrm{~mm}$ de espesor, soldadas al arco y cubierta con paños de red de poliamida torcida 24/210 de longitud de malla de $3,81 \mathrm{~cm}$ (1,5 pulgadas). La entrada o boca, ubicada en la pared lateral de la trampa a aproximadamente $20 \mathrm{~cm}$ del piso, fue confeccionada en forma rectangular de 30x10 cm, utilizando varillas de fierro de $0,6 \mathrm{~mm}$ de sección.

8. Piramidal pequeña con entrada circular (D2): Difiere del modelo anterior en la entrada o boca, la que fue confeccionada en forma de anillo de $25 \mathrm{~cm}$ de diámetro, utilizando varillas de fierro de $0,6 \mathrm{~mm}$ de sección.

9. Piramidal grande con entrada rectangular (E1): Construida de $100 \times 100 \mathrm{~cm}$ de base inferior, $60 \mathrm{~cm}$ de alto y 70x70 $\mathrm{cm}$ de base superior, con barras de fierro dulce de sección cuadrada de $14 \mathrm{~mm}$ de espesor, soldadas al arco y cubierta con paños de red de poliamida torcida $24 / 210$ de longitud de malla de $3,81 \mathrm{~cm}$ (1,5 pulgadas). La entrada o boca, ubicada en la pared lateral de la trampa a aproximadamente $20 \mathrm{~cm}$ del piso, fue confeccionada en forma rectangular de 30x10 cm, utilizando varillas de fierro de $0,6 \mathrm{~mm}$ de sección.
10. Piramidal grande con entrada circular (E2): Con el mismo diseño anterior, la entrada o boca fue confeccionada en forma circular de $25 \mathrm{~cm}$ de diámetro, utilizando varillas de fierro de $0,6 \mathrm{~mm}$ de sección.

11. Langostera con entrada en un extremo (FI): De estructura rectangular $(167 \times 80 \times 40 \mathrm{~cm})$, esta trampa fue construida con maderas locales; armazón de eucalipto (Eucalyptus sp.), forrada con varillas de maqui (Aristotelia maqui) en los costados, paredes laterales y división interior. La entrada o "buche" a cada cámara, en forma de cono, fue fabricada manualmente mediante el tejido de mallas de polietileno de dos pulgadas $(5,08 \mathrm{~cm})$ de longitud entre nudos. La entrada exterior se ubicó en un extremo de la trampa.

12. Langostera con entrada en la parte superior (GL): Con pequeñas diferencias en el diseño y dimensiones con respecto a la trampa FI $(135 \times 78 \times 35 \mathrm{~cm})$. En este caso particular, la entrada exterior se ubica en la parte superior de la trampa, sobre una de las cámaras.

Para el calado de las trampas se utilizó un orinque de polietileno torcido (PE) de 5 ó $6 \mathrm{~mm}$ de diámetro. Durante las experiencias se probó con el calado individual de las trampas y en forma de tena; en este último caso se utilizó un chicote de aproximadamente $30 \mathrm{~m}$ de longitud, del mismo material para unir la trampa al orinque, procurando que se emplearan diseños diferentes en cada línea, distanciadas alrededor de $100 \mathrm{~m}$ una de otra. El empleo de un chicote de esa longitud permite afianzar la línea madre mientras se vira la respectiva trampa.

Una vez caladas las trampas en cada estación de trabajo, se colocó una boya rígida a mediagua de 12 $\mathrm{cm}$ de diámetro y otra rígida de $35 \mathrm{~cm}$ de diámetro en superficie y flotadores Vinycom (4) de $5 \mathrm{~kg}$ de boyantes a modo de baticulo, con el fin de servir estas últimas de resguardo y demarcación de la trampa o tena. Las trampas langosteras fueron lastradas con piedras de aproximadamente $6 \mathrm{~kg}$, evitando con esto su desplazamiento por el fondo marino, mientras que los restantes diseño no lo requirieron por el peso aportado por el fierro utilizado en su armazón. Como carnada de las trampas se utilizó alrededor de $2 \mathrm{~kg}$ de especies ícticas locales, capturadas previamente al calado de los aparejos.

En cada salida se llevó registro de la fecha, posición geográfica, profundidad de trabajo, tipo de carnada utilizada, diseño de las trampas y captura en número obtenida en cada unidad experimental 
(trampa). Como medida del rendimiento se calculó la captura por unidad de esfuerzo (CPUE) por especie, separadamente por transecta, tipo de trampa empleado y profundidad de muestreo.

Además, se llevó control in situ del sexo, la longitud del caparazón, utilizando para ello un pie de metro con precisión de $\pm 1 \mathrm{~mm}$ y peso total individual de los ejemplares, este último determinado mediante un dinamómetro. Paralelamente, se obtuvieron muestras de los crustáceos y de los peces capturados, los que fueron conservados en formalina para su identificación taxonómica.

\section{RESULTADOS}

\section{Aspectos generales}

Las operaciones de pesca con trampas en torno a las islas Robinson Crusoe y Santa Clara, se efectuaron entre el 11 de noviembre de 1996 y el 23 de marzo de 1997. En dicho período se realizó un total de 71 salidas, en las que se caló un total de 241 trampas en 36 estaciones de pesca (Fig. 1). Del total de aparejos utilizados, 80 fueron calados en la transecta 1 (Puerto Francés), 67 en la transecta 2 (Islote Juanango), 45 en la transecta 3 (Bahía El Padre) y 49 en la transecta 4 (El Verdugo).

Iniciadas las faenas, se observó que los diseños de trampa que se destacaban por obtener mayores capturas fueron las troncocónicas (A1 y A2) y las tetragonales chicas (B1 y B2). Así, también, después de un cierto número de pruebas por problemas de operación se decidió eliminar el uso de las trampas de diseño tetragonal grande ( $\mathrm{C} 1$ y $\mathrm{C} 2)$ y piramidal grande (E1 y E2), debido principalmente a su gran tamaño, que dificultaba transportarlas y manejarlas a bordo de la embarcación, así como también porque generaban menores capturas que sus similares de menor tamaño.

El régimen original determinado para la operación de pesca exploratoria con trampas consideraba un período de reposo del aparejo de $24 \mathrm{hr}$. Sin embargo, debido a las características oceánicas de estas islas y las condiciones meteorológicas y de corrientes imperantes, este lapso se extendió en algunas oportunidades a 48, 72, 96 e incluso en forma excepcional a $120 \mathrm{hr}$.

Con los diferentes diseños de trampa empleados, se capturaron cuatro especies de crustáceos (Retamal y Arana, 2000) y cinco especies de peces. La captura total obtenida en la exploración fue
$380,14 \mathrm{~kg}$, representando el primer grupo el $95 \%$ de dicha captura.

Los crustáceos capturados correspondieron a: cangrejo dorado (Chaceon chilensis), langosta de Juan Fernández (Jasus frontalis), centolla de Juan Fernández (Paromola rathbuni) y jaiba remadora (Ovalipes trimaculatus de Haan, 1833). La captura total obtenida de estos recursos fue de $361 \mathrm{~kg}$, que correspondió a 432 individuos (Tabla 1). El recurso que presentó el mayor porcentaje respecto al peso total retenido por las trampas fue el cangrejo dorado $(72,2 \%)$, seguido por la centolla de Juan Fernández $(23,6 \%)$, jaiba remadora $(3,1 \%)$ y langosta de Juan Fernández (1,1\%).

La fauna acompañante asociada a la captura de crustáceos estuvo constituida por cinco especies: ribaldo (Mora moro Risso, 1810), anguila de profundidad (Bassanago nielseni Karmovskaya, 1990), morena (Gymnothorax porphyreus Guichenot, 1848), chancharro (Helicolenus lengerichi Norman, 1937) y pulpo (Octopus vulgaris Cuvier, 1797). La captura de esta fauna acompañante tuvo volúmenes muy reducidos, destacándose el pulpo con sólo 9 ejemplares (Tabla 2). Cabe señalar que cada especie fue capturada en una sola transecta, excepto la morena que apareció en las transectas 3 (Bahía El Padre) y 4 (Islote El Verdugo).

Con respecto al tipo de trampa empleado, se observa que el modelo troncocónico obtuvo mayor captura de fauna acompañante que los restantes, ya que se capturó con dicho modelo 12 de los 20 ejemplares capturados en toda la experiencia. Por otro lado, la mayoría de las capturas se obtuvieron hasta los $300 \mathrm{~m}$, con excepción de un ribaldo capturado a $800 \mathrm{~m}$ (Tabla 2).

De la información registrada en los lances exploratorios es posible destacar los siguientes aspectos respecto a las cuatro especies de crustáceos capturadas alrededor de las islas Robinson Crusoe y Santa Clara:

\section{Jaiba remadora (Ovalipes trimaculatus)}

De acuerdo a los datos resultantes de la pesca exploratoria con trampas, la jaiba remadora se encontró sólo en una de las transectas (I. Juanango), donde se capturó un total de 29 ejemplares, con $11,07 \mathrm{~kg}$ de peso total (Tabla 1). El rendimiento obtenido por trampa en el estrato en que se encontró esta especie, en términos de individuos y de peso capturado por unidad de pesca, fue de 0,76 ind./trampa y $0,29 \mathrm{~kg} /$ trampa, respectivamente. El peso promedio de estos organismos fue de $382 \mathrm{~g}$. 
Tabla 1. Captura y rendimiento de crustáceos capturados en la pesca exploratoria según transecta, tipo de trampa y profundidad de calado.

Table 1. Catch and yields of crustaceans obtained by transect, trap type and setting depth.

\begin{tabular}{|c|c|c|c|c|c|c|c|c|c|c|c|c|c|c|c|c|c|c|c|c|c|c|}
\hline & & \multirow{3}{*}{$\begin{array}{c}\mathrm{N}^{\mathrm{N}} \\
\text { trampas } \\
\text { caladas }\end{array}$} & \multicolumn{5}{|c|}{ Jaiba remadora } & \multicolumn{5}{|c|}{ Langosta de Juan Fernández } & \multicolumn{5}{|c|}{ Centolla de Juan Fernández } & \multicolumn{5}{|c|}{ Cangrejo dorado } \\
\hline & & & \multicolumn{2}{|c|}{ Captura } & \multicolumn{2}{|c|}{ CPUE } & \multirow{2}{*}{$\begin{array}{c}\text { Peso } \\
\text { promedio } \\
\text { individual } \\
(\mathrm{kg})\end{array}$} & \multicolumn{2}{|c|}{ Captura } & \multicolumn{2}{|c|}{ CPUE } & \multirow{2}{*}{$\begin{array}{l}\text { Peso } \\
\text { promedio } \\
\text { individual } \\
(\mathbf{k g})\end{array}$} & \multicolumn{2}{|c|}{ Captura } & \multicolumn{2}{|c|}{ CPUE } & \multirow{2}{*}{$\begin{array}{c}\text { Peso } \\
\text { promedio } \\
\text { individual } \\
\text { (kg) }\end{array}$} & \multicolumn{2}{|c|}{ Captura } & \multicolumn{2}{|c|}{ CPUE } & \multirow[b]{2}{*}{$\begin{array}{c}\text { Peso } \\
\text { promedio } \\
\text { individual } \\
\text { (kg) }\end{array}$} \\
\hline & & & & $\begin{array}{l}\text { Peso } \\
(\mathrm{kg})\end{array}$ & $\begin{array}{l}\text { ind / } \\
\text { trampa }\end{array}$ & $\begin{array}{c}\mathrm{kg} / \\
\text { trampa }\end{array}$ & & $\mathrm{N}^{2}$ & $\begin{array}{l}\text { Peso } \\
(\mathrm{kg})\end{array}$ & $\begin{array}{l}\text { ind / } \\
\text { trampa }\end{array}$ & $\begin{array}{c}\mathrm{kg} / \\
\text { trampa }\end{array}$ & & No & $\begin{array}{l}\text { Peso } \\
(\mathrm{kg})\end{array}$ & $\begin{array}{l}\text { ind I } \\
\text { trampa }\end{array}$ & $\begin{array}{c}\mathrm{kg} / \\
\text { trampa }\end{array}$ & & $\mathrm{N}^{\mathbf{2}}$ & $\begin{array}{l}\text { Peso } \\
(\mathbf{k g})\end{array}$ & $\begin{array}{c}\text { ind } l \\
\text { trampa }\end{array}$ & $\underset{\text { trampa }}{\mathrm{kg} /}$ & \\
\hline \multirow{3}{*}{ 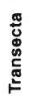 } & $\begin{array}{r}1 \text { (Pto. Francés) } \\
2 \text { (1. Juanango) }\end{array}$ & $\begin{array}{l}80 \\
67\end{array}$ & 29 & 11.07 & 0.43 & 0.17 & $\cdot \cdot \cdot \cdot 382$ & . & . & . & . & & $\begin{array}{l}21 \\
76\end{array}$ & $\begin{array}{l}17,75 \\
50,10\end{array}$ & 0,26 & $\begin{array}{l}0,22 \\
0,75\end{array}$ & $\begin{array}{l}0,845 \\
0.599\end{array}$ & 126 & 130,52 & 1.58 & 1,63 & 1,036 \\
\hline & $\begin{array}{l}\text { 2(1. Juanango) } \\
3 \text { (Ba. El Padre) }\end{array}$ & 45 & . & . & 0,45 & . & 0,082 & 2 & 1.08 & 0.04 & 0,02 & 0,540 & $\begin{array}{l}16 \\
35\end{array}$ & $\begin{array}{l}50,10 \\
11,65\end{array}$ & $\begin{array}{l}1,13 \\
0,78\end{array}$ & 0,75 & 0,659 & 42 & 42,95 & 0,63 & 0,64 & 1,023 \\
\hline & 4 (El Verdugo) & 49 & . & . & . & . & . & 5 & 2,72 & 0,10 & 0,06 & 0,540 & 6 & 5,80 & 0,12 & 0,26 & 0,333 & 49 & 46,81 & 1,09 & 1,04 & 0,955 \\
\hline \multirow{12}{*}{ 总 } & $A_{1}$ & 34 & 16 & 7.60 & 0.47 & 0.22 & 0.480 & - & & . & - & - & & 430 & & & & & & & & \\
\hline & A2 & 36 & . & . & . & . & . & . & . & . & . & . & 32 & 16,20 & 0.89 & 0.45 & 0,506 & 19 & 18,45 & 0.53 & $\begin{array}{l}0.83 \\
0.51\end{array}$ & $\begin{array}{l}1,047 \\
0,971\end{array}$ \\
\hline & B1 & 39 & & - & $\cdot$ & - & - & - & . & & & . & 6 & 3,40 & 0,15 & 0,09 & 0,567 & 65 & 64,19 & 1,67 & 1,65 & 0,988 \\
\hline & B2 & 36 & 1 & 0,20 & 0.03 & 0,01 & 0,200 & 5 & 2,59 & 0,14 & 0,07 & 0,520 & 24 & 11,00 & 0,67 & 0,31 & 0.458 & 49 & 50,23 & 1,36 & 1,40 & 1,025 \\
\hline & $\mathrm{C} 1$ & 8 & 1 & 0,20 & 0,13 & 0,03 & 0,200 & - & . & - & . & - & . & & . & . & . & 9 & 9,20 & 1,13 & 1,15 & 1,022 \\
\hline & $\mathrm{C} 2$ & 14 & 4 & 0.67 & 0.29 & 0.05 & 0,170 & 2 & 1,21 & 0,14 & 0,09 & 0,610 & 14 & 11,80 & 1,00 & 0,84 & 0,843 & 22 & 20,80 & 1.57 & 1.49 & 0,945 \\
\hline & D1 & 24 & 3 & 1,20 & 0,13 & 0,05 & 0,400 & . & & . & . & . & 11 & 8,10 & 0.46 & 0.34 & 0,736 & 28 & 30,26 & 1,17 & 1,26 & 1,081 \\
\hline & D2 & 22 & . & $\cdot$ & . & $\cdot$ & . & . & . & . & & . & 19 & 11,95 & 0,86 & 0,54 & 0,629 & 22 & 20,12 & 1,00 & 0,91 & 0,915 \\
\hline & E1 & 12 & 4 & 1,20 & 0.33 & 0.10 & 0,300 & . & . & . & . & . & 5 & 4,70 & 0,42 & 0,39 & 0,940 & 10 & 10,60 & 0.83 & 0,88 & 1,060 \\
\hline & E2 & 8 & . & $\cdot$ & $\cdot$ & $\cdot$ & · & . & . & . & . & . & 20 & 12,05 & 2.50 & 1.51 & 0.603 & 1 & 1,30 & 0,13 & 0,16 & 1,300 \\
\hline & $\mathrm{FI}$ & 4 & . & . & . & . & . & . & & & & . & 3 & 1,80 & 0,75 & 0,45 & 0,600 & 3 & 4,10 & 0,75 & 1,03 & 1,367 \\
\hline & GL & 4 & & - & & & . & $\cdot$ & . & $\cdot$ & . & . & - & $\cdot$ & $\cdot$ & $\cdot$ & · & 3 & 3,25 & 0,75 & 0,81 & 1,083 \\
\hline \multirow{11}{*}{ 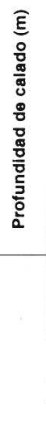 } & 50 & 38 & . & . & $\cdot$ & $\cdot$ & . & 6 & 3.27 & 0,16 & 0,09 & 0,550 & & . & $\cdot$ & . & . & . & . & . & . & . \\
\hline & 100 & 38 & 29 & 11,07 & 0.76 & 0.29 & 0,382 & 1 & 0,53 & 0,03 & 0,02 & 0,530 & 25 & 16,10 & 0,66 & 0.42 & 0,644 & 3 & 4,10 & 0,08 & 0,11 & 1,367 \\
\hline & 200 & 55 & . & . & $\cdot$ & . & · & . & . & . & $\cdot$ & . & 79 & 37,95 & 1,44 & 0,69 & 0,480 & 60 & 62,95 & 1,09 & 1,14 & 1,049 \\
\hline & 300 & 30 & . & & . & - & - & & . & & . & . & 34 & 31,25 & 1,13 & 1,04 & 0,919 & 80 & 83,52 & 2,67 & 2,78 & 1,044 \\
\hline & 400 & 21 & . & . & & - & - & - & . & . & - & . & . & . & $\cdot$ & . & . & 40 & 40,52 & 1,90 & 1,93 & 1,013 \\
\hline & 500 & 19 & . & . & . & . & . & . & & . & - & - & . & & . & . & . & 31 & 29,76 & 1,63 & 1,57 & 0,960 \\
\hline & 600 & 13 & - & & & & - & $\cdot$ & $\cdot$ & $\cdot$ & - & - & . & - & - & . & - & 23 & 20,78 & 1,77 & 1,60 & 0,903 \\
\hline & 800 & 17 & . & . & - & - & - & . & . & . & - & - & . & & - & . & . & 19 & 17,55 & 1,12 & 1,03 & 0,924 \\
\hline & 1000 & 10 & . & . & . & . & . & - & . & & - & . & . & . & - & . & . & 2 & 1,60 & 0,20 & 0.16 & 0,800 \\
\hline & Total & 241 & 29 & 11,07 & 0,12 & 0,05 & 0,382 & 7 & 3,80 & 0,03 & 0,02 & 0,540 & 138 & 85,30 & 0,57 & 0,35 & 0,618 & 258 & 260,78 & 1,07 & 1,08 & 1,011 \\
\hline & $\begin{array}{l}\text { A1: Troncocónica } \\
\text { A2: Troncocónica } \\
\text { B1: Tetragonal per }\end{array}$ & $\begin{array}{l}\text { nntrada rec } \\
\text { entrada cir } \\
\text { jueña entra }\end{array}$ & a recto & Igular & $\begin{array}{l}\text { B2: } \\
\text { C1: } \\
\text { C2: }\end{array}$ & $\begin{array}{l}\text { Etragonal } \\
\text { tragonal } \\
\text { tragonal }\end{array}$ & $\begin{array}{l}\text { ueña entrada } \\
\text { nde entrada } \\
\text { dde entrada }\end{array}$ & $\begin{array}{l}\text { tangul } \\
\text { ular }\end{array}$ & & $\begin{array}{l}\text { D1: } P \\
\text { D2: } P \\
\text { E1: } P\end{array}$ & $\begin{array}{l}\text { Siramidata } \\
\text { itramidal } \\
\text { iiramidal }\end{array}$ & $\begin{array}{l}\text { equeñana entratra } \\
\text { equenta } \\
\text { rande entrad }\end{array}$ & $\begin{array}{l}\text { a rectar } \\
\text { a circulis } \\
\text { rectang }\end{array}$ & & $\begin{array}{l}\text { E2: } \\
\text { Fl: } \\
\text { GL: }\end{array}$ & $\begin{array}{l}\text { goostiter } \\
\text { goster }\end{array}$ & $\begin{array}{l}\text { Jaentrada } \\
\text { rade en un } \\
\text { adda parte }\end{array}$ & & & & & \\
\hline
\end{tabular}


Tabla 2. Fauna acompañante obtenida en la pesca exploratoria según transecta, tipo de trampa y profundidad de calado.

Table 2. Bycatch obtained by transect, trap type and setting depth.

Tabla 2. Fauna acompañante obtenida en la pesca exploratoria según transecta, tipo de trampa y profundidad de calado.

Table 2. Bycatch obtained by transect, trap type and setting depth.

\begin{tabular}{|c|c|c|c|c|c|c|c|c|c|c|c|c|}
\hline & & \multirow{3}{*}{$\begin{array}{l}\text { № trampas } \\
\text { caladas }\end{array}$} & \multicolumn{10}{|c|}{ Recurso capturado } \\
\hline & & & \multicolumn{2}{|c|}{ Pulpo } & \multicolumn{2}{|c|}{ Ribaldo } & \multicolumn{2}{|c|}{ Anguila de profundidad } & \multicolumn{2}{|c|}{ Morena } & \multicolumn{2}{|c|}{ Chancharro } \\
\hline & & & & $\begin{array}{c}\text { Peso } \\
\text { (kg) }\end{array}$ & $\mathrm{N}^{2}$ & $\begin{array}{l}\text { Peso } \\
\text { (kg) }\end{array}$ & & $\begin{array}{c}\text { Peso } \\
(\mathrm{kg})\end{array}$ & No & $\begin{array}{c}\text { Peso } \\
\text { (kg) }\end{array}$ & № & $\begin{array}{r}\text { Peso } \\
\text { (kg) }\end{array}$ \\
\hline 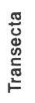 & $\begin{array}{c}1 \text { (Pto. Francés) } \\
2 \text { (I. Juanango) } \\
3 \text { (Ba. El Padre) } \\
4 \text { (EI Verdugo) }\end{array}$ & $\begin{array}{l}80 \\
67 \\
45 \\
49\end{array}$ & - & $\begin{array}{c}- \\
- \\
- \\
2.54\end{array}$ & $\begin{array}{l}- \\
- \\
1 \\
-\end{array}$ & $\begin{array}{c}- \\
- \\
1,40 \\
-\end{array}$ & $\begin{array}{l}4 \\
- \\
-\end{array}$ & $\begin{array}{c}5,00 \\
- \\
- \\
-\end{array}$ & $\begin{array}{l}- \\
- \\
3 \\
2\end{array}$ & $\begin{array}{c}- \\
- \\
5,95 \\
3,70\end{array}$ & $\begin{array}{l}1 \\
- \\
- \\
-\end{array}$ & $\begin{array}{c}0,60 \\
- \\
-\end{array}$ \\
\hline 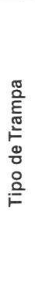 & $\begin{array}{l}\text { A1 } \\
\text { A2 } \\
\text { B1 } \\
\text { B2 } \\
\text { C1 } \\
\text { C2 } \\
\text { D1 } \\
\text { D2 } \\
\text { E1 } \\
\text { E2 } \\
\text { F1 } \\
\text { GL }\end{array}$ & $\begin{array}{c}34 \\
36 \\
39 \\
36 \\
8 \\
14 \\
24 \\
22 \\
12 \\
8 \\
4 \\
4\end{array}$ & $\begin{array}{l}5 \\
- \\
1 \\
- \\
- \\
- \\
1 \\
2 \\
- \\
- \\
-\end{array}$ & $\begin{array}{c}1,50 \\
- \\
0,24 \\
- \\
- \\
- \\
0,30 \\
0,50 \\
- \\
- \\
- \\
-\end{array}$ & $\begin{array}{l}1 \\
- \\
- \\
- \\
- \\
- \\
- \\
-\end{array}$ & $\begin{array}{c}1,40 \\
- \\
- \\
- \\
- \\
- \\
- \\
- \\
- \\
- \\
- \\
-\end{array}$ & $\begin{array}{l}- \\
3 \\
- \\
- \\
- \\
- \\
- \\
- \\
- \\
1 \\
-\end{array}$ & $\begin{array}{c}- \\
3,50 \\
- \\
- \\
- \\
- \\
- \\
- \\
- \\
1,50 \\
- \\
-\end{array}$ & $\begin{array}{l}1 \\
2 \\
- \\
- \\
- \\
- \\
2 \\
- \\
- \\
-\end{array}$ & $\begin{array}{c}4,00 \\
3,70 \\
- \\
- \\
- \\
- \\
- \\
1,95 \\
- \\
- \\
- \\
-\end{array}$ & $\begin{array}{l}- \\
- \\
- \\
- \\
- \\
- \\
- \\
- \\
-\end{array}$ & $\begin{array}{c}- \\
- \\
- \\
- \\
0.60 \\
- \\
- \\
- \\
- \\
-\end{array}$ \\
\hline 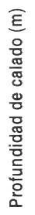 & $\begin{array}{c}50 \\
100 \\
200 \\
300 \\
400 \\
500 \\
600 \\
800 \\
1000\end{array}$ & $\begin{array}{l}38 \\
38 \\
55 \\
30 \\
21 \\
19 \\
13 \\
17 \\
10\end{array}$ & $\begin{array}{l}- \\
9 \\
- \\
- \\
- \\
- \\
-\end{array}$ & $\begin{array}{c}- \\
2,54 \\
- \\
- \\
- \\
- \\
- \\
- \\
-\end{array}$ & $\begin{array}{l}- \\
- \\
- \\
- \\
- \\
1 \\
-\end{array}$ & $\begin{array}{c}- \\
- \\
- \\
- \\
- \\
- \\
- \\
1,40 \\
-\end{array}$ & $\begin{array}{l}- \\
1 \\
3 \\
- \\
- \\
- \\
- \\
- \\
-\end{array}$ & $\begin{array}{c}\cdot \\
1,50 \\
3,50 \\
- \\
- \\
- \\
- \\
-\end{array}$ & $\begin{array}{l}3 \\
- \\
- \\
2 \\
- \\
- \\
- \\
- \\
-\end{array}$ & $\begin{array}{c}5,95 \\
- \\
- \\
3,70 \\
- \\
- \\
- \\
- \\
-\end{array}$ & $\begin{array}{l}- \\
- \\
1 \\
- \\
- \\
- \\
- \\
-\end{array}$ & $\begin{array}{c}- \\
- \\
0.60 \\
- \\
- \\
- \\
- \\
- \\
-\end{array}$ \\
\hline & Total & 241 & 9 & 2,54 & 1 & 1,40 & 4 & 5,00 & 5 & 9,65 & 1 & 0,60 \\
\hline & $\begin{array}{l}\text { A1: Troncocónica e } \\
\text { A2: Troncocónica e } \\
\text { B1: Tetragonal peq } \\
\text { B2: Tetragonal peq } \\
\text { C1: Tetragonal gran } \\
\text { C2: Tetragonal gran }\end{array}$ & $\begin{array}{l}\text { da rectangula } \\
\text { da circular } \\
\text { a entrada rect } \\
\text { a entrada circi } \\
\text { entrada rectar } \\
\text { entrada circul }\end{array}$ & lar & & & $\begin{array}{l}\text { D1: } \\
\text { D2: } \\
\text { E1: } \\
\text { E2: } \\
\text { FI: } \\
\text { GL: }\end{array}$ & $\begin{array}{l}\text { Piramidal peq } \\
\text { iramidal peq } \\
\text { iramidal grar } \\
\text { iramidal grar } \\
\text { angostera en } \\
\text { angostera en }\end{array}$ & $\begin{array}{l}\text { eña entrada re } \\
\text { eña entrada cir } \\
\text { le entrada recta } \\
\text { le entrada circu } \\
\text { ada en un extr } \\
\text { ada parte supe }\end{array}$ & $\begin{array}{l}\text { igula } \\
\text { ular }\end{array}$ & & & \\
\hline
\end{tabular}

De las trampas utilizadas destaca la alta retención lograda con la trampa troncocónica con entrada rectangular (A1), la cual registró el 55\% del total de individuos capturados (Tabla 1). Con respecto a la distribución batimétrica de esta especie, los datos fueron concluyentes, ubicándola exclusivamente en el veril de $100 \mathrm{~m}$.

Otro aspecto relevante es que del total de ejemplares capturados, el 89,7\% correspondió a machos. Las longitudes cefalotorácicas estuvieron comprendidas entre 6,9 y $8,4 \mathrm{~cm}$, con medias de 8,1 y $7,2 \mathrm{~cm}$ de longitud del caparazón en machos y hembras, respectivamente (Fig. 4).

\section{Langosta de Juan Fernández (Jasus frontalis)}

Esta especie fue la que registró la menor captura con sólo 7 individuos, de los cuales dos se extrajeron en la transecta 3 (Bahía El Padre) y 5 en la transecta 4 (El Verdugo) (Tabla 1). Los diseños de trampa que capturaron esta especie fueron la tetragonal pequeña y grande, ambas con entrada circular (B2 y C2). Como era de esperar, el estrato de profundidad en que se encontró este recurso fue entre 50 y $100 \mathrm{~m}$ (Tabla 1), rango en el cual es extraído este recurso por los pescadores de estas islas. En dicho rango se obtuvo un rendimiento de 0,09 ind./ trampa y $0,05 \mathrm{~kg} /$ trampa. Cabe señalar que las hembras se presentaron en mayor proporción $(85,7 \%)$ que los machos.

\section{Centolla de Juan Fernández (Paromola rathbuni)}

La centolla de Juan Fernández registró una captura total de 138 ejemplares, con un peso de $85,3 \mathrm{~kg}$ y un peso promedio individual de $618 \mathrm{~g}$. Dichas capturas fueron obtenidas en forma indiferenciada por 
la mayoría de los diseños de trampa, con un rendimiento promedio general en el estrato en que se encontró esta especie de 1,12 ind./ trampa y 0,69 kg/trampa (Tabla 1 ).

En esta especie se determinó una significativa preponderancia de machos $(70,4 \%)$. Las longitudes cefalotorácicas de los ejemplares capturados estuvieron comprendidas entre 6,9 y 13,0 cm (media $=10,1 \mathrm{~cm}$ en machos y media $=9,0 \mathrm{~cm}$ en hembras) (Fig. 4).

Este recurso se encontró entre los $100 \mathrm{y}$ $300 \mathrm{~m}$, en todas las transectas consideradas alrededor de estas islas. Cabe destacar la captura obtenida en el veril de $200 \mathrm{~m}$, con 79 individuos, lo que constituye el $57 \%$ del total capturado (Tabla 1).

\section{Cangrejo dorado (Chaceon chilensis)}

Como se mencionó anteriormente, de las especies capturadas, la que llamó más la atención fue la aparición del cangrejo dorado (Fig. 5). Los resultados obtenidos por transecta, indican capturas de este crustáceo distribuidas alrededor de toda la isla, con la mayor captura en la transecta frente a Puerto Francés (1,63 kg/trampa) y la menor frente al islote Juanango $(0,64 \mathrm{~kg} / \mathrm{tram}$ pa) (Tabla 1).

La proporción sexual del cangrejo dorado alrededor de las islas Robinson Crusoe y Santa Clara presentó una significativa mayoría de machos $(98,1 \%)$. Los ejemplares presentaron tallas entre 8,6 y $14,0 \mathrm{~cm}$ de longitud cefalotorácica, con una media de $12,3 \mathrm{~cm}$ en machos y $10,1 \mathrm{~cm}$ en hembras (Fig. 4).

Las trampas de diseño troncocónico (A1 y A2), tetragonal pequeña (B1 y B2) y piramidal pequeña (D1 y D2) obtuvieron las capturas más numerosas con 210 de un total de 258 ejemplares capturados. El peso promedio alcanzó los 1011 g, registrándose por lo tanto, rendimientos muy similares en términos de individuos por trampa y kilogramos por trampa $(1,27$ y 1,28 respectivamente).

Otro aspecto de interés, fue el amplio rango de distribución encontrado en esta especie, abarcando casi todo el rango de profundidad estudiado (100 a $1000 \mathrm{~m}$ ). Las

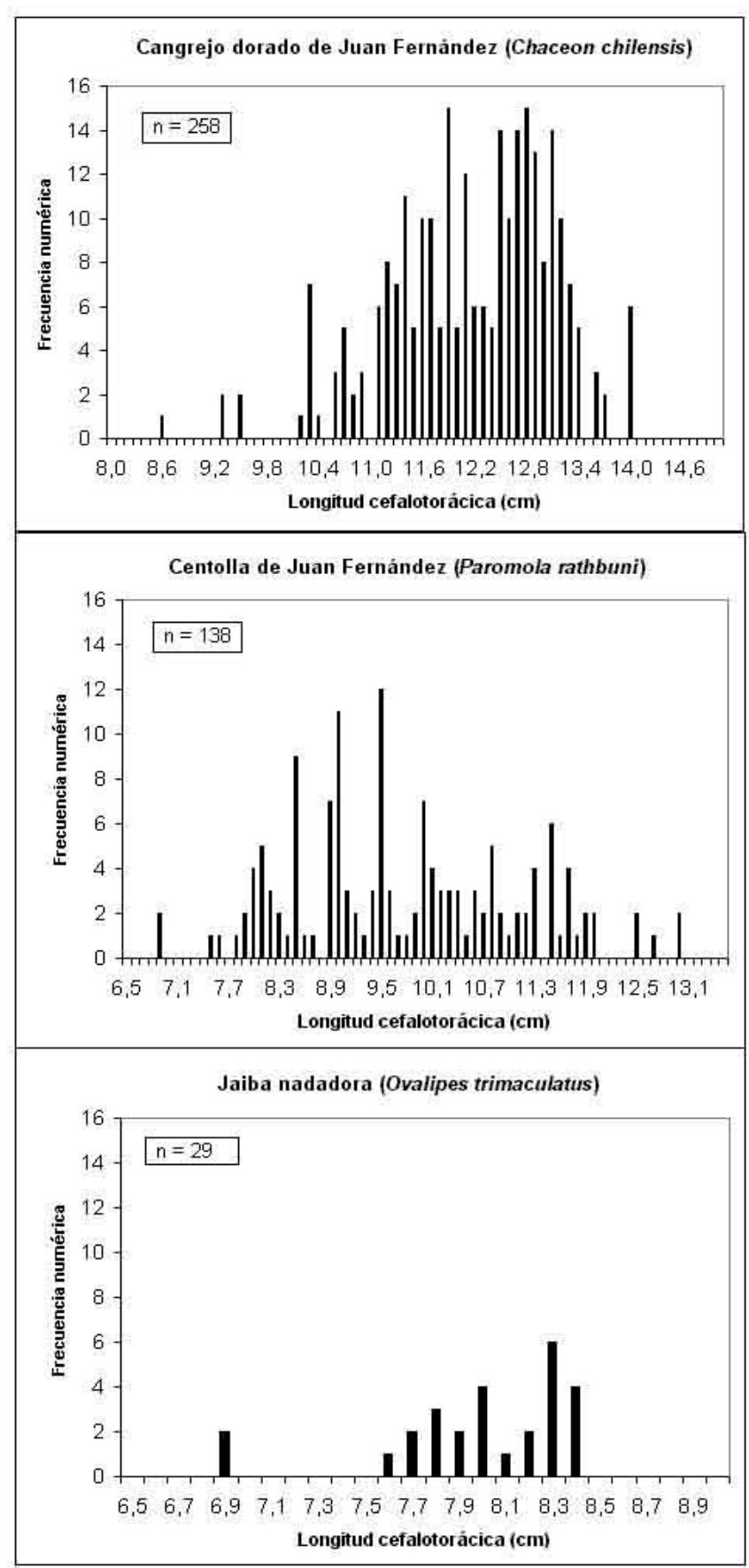

Figura 4. Distribuciones de frecuencias de tallas de tres especies de crustáceos capturados en la pesca exploratoria.

Figure 4. Length frequency distributions for the three crustacean species caught during the fishing survey. 


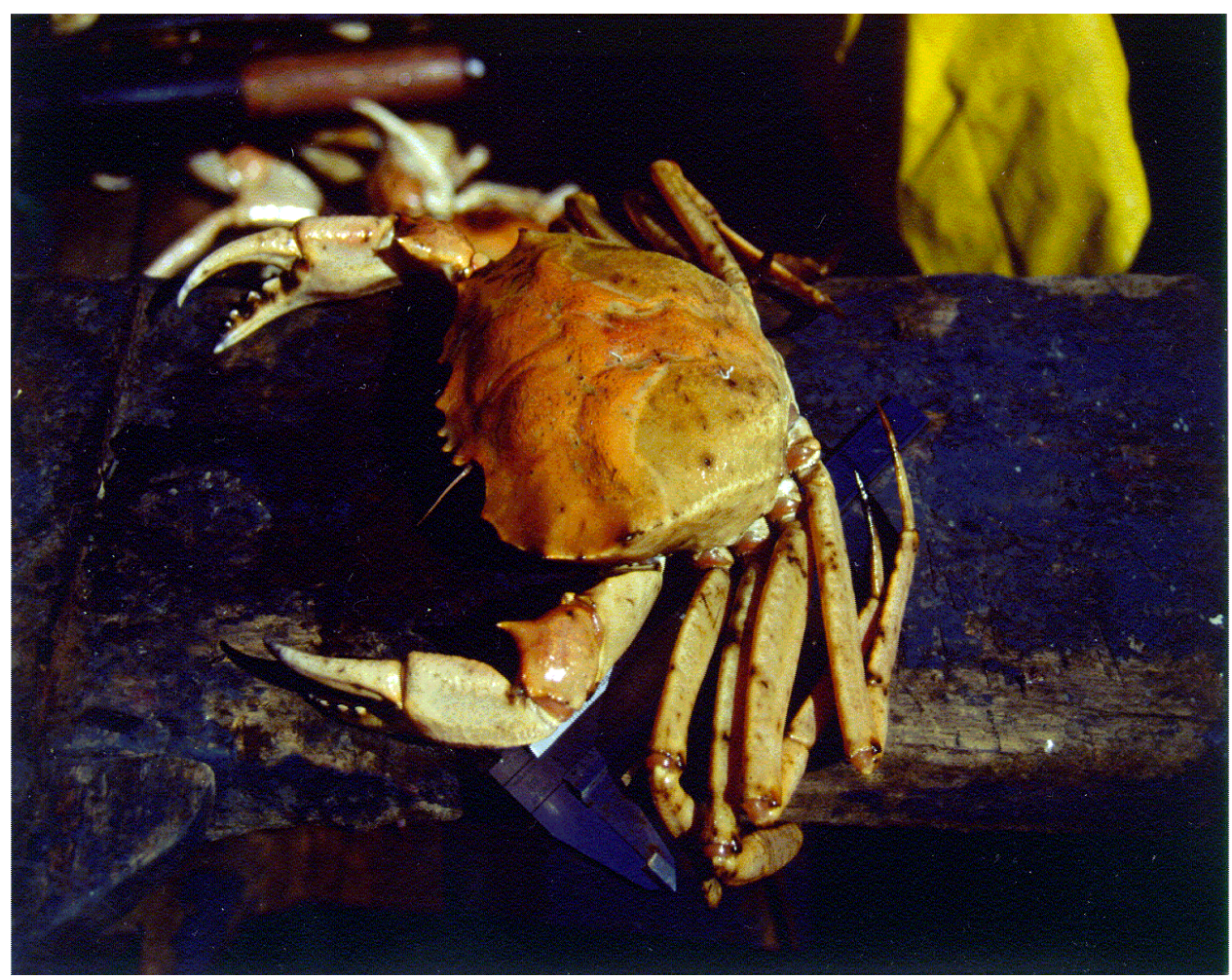

Figura 5. Cangrejo dorado de Juan Fernández (Chaceon chilensis), macho.

Figure 5. Juan Fernandez golden crab (Chaceon chilensis), male.

capturas en profundidad presentaron valores que aumentaron hasta $300 \mathrm{~m}$ y luego disminuyeron progresivamente hasta llegar a $1000 \mathrm{~m}$ (Tabla 2).

\section{DISCUSIÓN}

Las principales actividades que se efectúan en una fase primaria de desarrollo pesquero, es la búsqueda de recursos susceptibles de ser explotados, la evaluación de los efectivos disponibles y la investigación básica de las especies más relevantes (Williams, 1964; Troadec, 1978). Como primera opción, se recurre a la realización de faenas de pesca exploratoria, en la que por lo general se emplea un arte de pesca cuyas características estructurales y operativas no varíen, de manera que se determine con éste la variabilidad intrínseca del o de los recursos existentes en el lugar (Gneri, 1966). Así, la pesca exploratoria tiene la ventaja de aportar con información que puede ser utilizada en forma directa en el desarrollo de pesquerías comerciales (Gulland, 1970). Por otra parte, para realizar dichas labores se requiere conocer, aunque sea en forma aproximada, cuál es el arte y al mismo tiempo las características del mismo que son más apropiadas para hacer la pesca exploratoria, ya que de lo contrario se podría obtener un resultado erróneo respecto a la presencia de determinados recursos o los rendimientos resultarían subestimados a los que se obtendrían con otros artes. De allí nace la imprescindible necesidad de comparar el desempeño de diferentes artes en simultaneidad de espacio y tiempo. En este último caso, se está ante el concepto de pesca experimental, en el cual ahora la población se considera como constante y las capturas obtenidas con los diferentes diseños en prueba revelan las diferencias propias de cada uno de ellos (Holt, 1956).

De allí que cuando se desea conocer la composición faunística en una región parcial o totalmente desconocida, en que por la misma razón se dispone de escaso conocimiento acerca del comportamiento de los recursos que van a ser capturados, a la vez que se requiere determinar su potencial como recurso pesquero, la opción más conveniente es utilizar simultáneamente un cierto número de artes de pesca de diferente diseño o características, de modo 
de efectuar al mismo tiempo faenas de pesca exploratoria y experimental. De esta manera, es posible determinar, a lo menos en una primera aproximación, el aparejo de pesca más conveniente para la extracción de las especies que se presentan como promisorias, a la vez que se proporcionan estimados próximos a los que se obtendrían al explotar los recursos que se investigan (Troadec, 1978).

En el caso de los crustáceos, la atracción, ingreso y retención de estos organismos en las trampas utilizadas para su captura depende de diversos factores, entre ellos, ambientales, fisiológicos y de comportamiento asociado a las características de las trampas (Krouse, 1989). Entre estos últimos, destacan, principalmente: a) el diseño de la trampa, como ser la forma y el tamaño de las mismas; b) la luz de las mallas o la separación de los listones que componen sus paredes; c) la forma, tamaño y localización de las entradas, respecto a la ubicación de la carnada; d) el empleo de mecanismos anti-escapes o que dificulten la salida de los ejemplares que ya han ingresado; d) la utilización de dispositivos empleados en algunos casos para facilitar el escape de organismos de pequeño tamaño; y, e) de las carnadas utilizadas, siendo relevante la capacidad de atracción que tenga sobre la especie objetivo y la duración de su efecto en relación al período de reposo.

Dada la escasa información disponible sobre qué recursos se podrían encontrar hasta los $1000 \mathrm{~m}$ de profundidad, la investigación realizada en el archipiélago de Juan Fernández se planificó considerando la utilización de diversos tipos de trampas. Con esto se pretendió determinar las posibles especies que se podrían explotar y las ventajas comparativas entre los distintos diseños, respecto a su utilización en embarcaciones artesanales de pequeño tamaño donde las faenas se realizan principalmente en forma manual.

Uno de los principales aspectos resultantes de la presente campaña de pesca fue la baja cantidad de especies encontradas, así como la presencia de alguna de ellas en lugares o estratos de profundidad claramente diferenciados, con escasa sobreposición entre ellas. Así, la langosta se capturó entre 50 y $100 \mathrm{~m}$, mientras que la jaiba remadora se encontró únicamente en el veril de los $100 \mathrm{~m}$. Por otro lado, entre los 100 y $300 \mathrm{~m}$ se distribuye la centolla, especie que aparece en forma conjunta con el cangrejo dorado principalmente en faenas realizadas entre 200 y 300 m. De los $300 \mathrm{~m}$ en adelante, este último recurso fue obtenido sin fauna asociada, caracterís- tica que se mantiene hasta los $1000 \mathrm{~m}$, máxima profundidad a la que fueron calados los aparejos. Cabe mencionar que un resultado semejante se obtuvo al realizar faenas de pesca exploratorias de peces en estos mismos fondos, utilizando en este caso espineles de profundidad tanto verticales como horizontales, encontrándose igualmente un escaso número de especies, notoria estratificación en la distribución de las mismas y bajos rendimientos (Arana y Vega, 2000). Esto último podría explicar igualmente la baja captura de fauna acompañante obtenida en las faenas realizadas con trampas.

La baja cantidad de ejemplares obtenida de Jasus frontalis, se puede explicar por el reducido número de trampas caladas en el estrato en que se distribuye esta especie, así como por el hecho de haberse calado las trampas en estaciones predefinidas sobre transectas dispuestas en forma perpendicular a la costa y no en lugares o caladeros en que se conoce la presencia de este crustáceo. Como es sabido, esta especie habita principalmente en zonas rocosas, con grietas y cuevas donde se refugian las langostas, lugares que son conocidos y utilizados por los pescadores para calar en ellos las trampas (Arana, 1987).

Los resultados obtenidos señalan al cangrejo dorado (C. chilensis) como el principal recurso carcinológico presente en aguas profundas en torno a las islas Robinson Crusoe y Santa Clara (Fig. 5). Este recurso no es desconocido por los pescadores de langosta, ya que quienes operan tanto en las islas Robinson Crusoe y Santa Clara, así como aquellos que se trasladan esporádicamente a las islas Desventuradas (islas San Félix y San Ambrosio), suelen capturar este cangrejo en calidad de fauna acompañante de la langosta, los que usualmente son consumidos por los tripulantes de las embarcaciones que los pescan. Estas capturas ocurren generalmente cuando los aparejos se desplazan del lugar donde fueron colocados sobre el fondo o son calados erróneamente a mayor profundidad ( $>200 \mathrm{~m}$ ). Anteriormente también se han capturado individuos de esta especie en pescas exploratorias realizadas en el cordón submarino de Juan Fernández (Conan, 1975), en la cordillera de Nazca, en faenas dirigidas a la pesca de la langosta enana o dalmacita (Projasus bahamondei George, 1976) (Arana y Venturini, 1991) y en algunas oportunidades en arrastres realizados en aguas profundas del margen continental de Chile (Báez y Andrade, 1977).

Los rendimientos promedio totales obtenidos de C. chilensis en la presente pesca exploratoria y ex- 
perimental fueron de 1,07 ind./trampa o $1,08 \mathrm{~kg} /$ trampa. Entre los diseños de trampa utilizados, el modelo que obtuvo mejor rendimiento en la captura del cangrejo dorado (1,67 ind./trampa y $1,65 \mathrm{~kg} /$ trampa) fue el tetragonal pequeño con entrada rectangular (B1), el cual además presentó la ventaja de resultar de fácil manipulación debido básicamente a su pequeño tamaño y forma. El otro tipo de trampa que generó buen rendimiento fue el diseño tetragonal grande con entrada circular (C2). En general, los modelos tetragonales pequeños (B1 y B2) $\mathrm{y}$ tetragonales grandes $(\mathrm{C} 1$ y $\mathrm{C} 2)$ obtuvieron mayor rendimiento que el resto de los diseños empleados (>1 ind. ó $1 \mathrm{~kg}$ por trampa).

Con respecto a la profundidad de calado, el veril de $300 \mathrm{~m}$ fue el estrato de profundidad en el que se registraron las mayores capturas, con rendimientos globales de 2,67 ind./trampa o 2,78 kg/trampa. Bajo esta profundidad y hasta $800 \mathrm{~m}$ los rendimientos fueron similares, entre 1,12 y 1,90 ind./trampa y 1,03 y $1,93 \mathrm{~kg} /$ trampa. Las diferencias en los rendimientos obtenidos en las diferentes transectas se explica de acuerdo al número y tipo de aparejos utilizados en cada una de ellas en la profundidad en que se encuentra la mayor abundancia de este recurso.

Se debe tener presente que la investigación se planificó con el objetivo principal de determinar la presencia de recursos de interés pesquero y no a definir en esta etapa el aparejo más adecuado para su extracción como tampoco el determinar áreas de concentración de las especies que presentaron la mayor abundancia. Por esta razón, en un proyecto dirigido a determinar el aparejo más adecuado para la explotación de este cangrejo, en las áreas en las que se concentra este crustáceo, con toda seguridad se obtendrían rendimientos muy superiores a los determinados en esta oportunidad.

No obstante lo anterior, tanto por el nivel de abundancia, como por el amplio rango batimétrico en el que se distribuye el cangrejo dorado es posible catalogarlo como el recurso que presenta las mejores perspectivas para ser considerado en el futuro para ser extraído y comercializado en estas islas. En el caso particular de la centolla de Juan Fernández, si bien se encontró con una abundancia relativa semejante al cangrejo dorado, la poca cantidad de carne y baja calidad de la misma la hace poco atractiva para ser extraída comercialmente. En cambio, $C$. chilensis presenta como características relevantes gran tamaño y peso, que se traduce en abundante carne, con buena textura y sabor. Cabe destacar, además, que esta especie es conocida en los mercados internacionales, siendo explotados cangrejos de esta familia en diversos lugares del mundo. Por ejemplo, en América del Sur se extrae desde hace algunos años el cangrejo rojo (Chaceon notialis Manning y Holthuis, 1989), en aguas jurisdiccionales del sur de Brasil y Uruguay (Dawson y Weber, 1991; FAO, 1996).

Aunque de carácter preliminar, los resultados obtenidos hasta ahora, despiertan interés en profundizar el estudio de este cangrejo. En especial se debe determinar el diseño de trampa con el cual se obtengan los más altos rendimientos, evaluar la cantidad que es factible de extraer anualmente, los costos de operación que implicaría su explotación y la aceptación y precio de comercialización de este recurso, tanto a nivel nacional como en el extranjero. Sobre la base de estos antecedentes se podría determinar la factibilidad de desarrollar a nivel artesanal la pesquería de $C$. chilensis, ya sea en forma alternativa o complementaria a la explotación de la langosta de Juan Fernández.

\section{AGRADECIMIENTOS}

El autor destaca la colaboración brindada por las tripulaciones de las chalupas de alta mar que participaron en la realización de la presente investigación. Así, también, agradece muy especialmente al Dr. Eduardo de la Hoz U. (Q.E.P.D.), de la Universidad Católica de Valparaíso, por su aporte en la identificación taxonómica de los peces y al Profesor Sr. Marco Antonio Retamal R., de la Universidad de Concepción, por la identificación de los crustáceos capturados en las trampas. Igualmente, el autor reconoce los constructivos aportes realizados por tres evaluadores anónimos que revisaron el artículo original.

\section{REFERENCIAS}

Arana, P. 1987. Perspectivas históricas y proyecciones de la actividad pesquera realizada en el archipiélago de Juan Fernández, Chile. En: J.C. Castilla (ed.). Islas Oceánicas Chilenas: Conocimiento Científico y Necesidades de Investigaciones, Edic. Univ. Católica de Chile, pp. 319-353. 
Arana, P. y R. Vega. 2000. Pesca exploratoria con espineles en aguas profundas en torno a la isla Robinson Crusoe (Archipiélago de Juan Fernández), Chile. Invest. Mar., Valparaíso, 28: 219-230.

Arana, P. y V. Venturini. 1991. Investigaciones biológico-pesqueras de crustáceos en la cordillera submarina de Nazca (Océano Pacífico Suroriental). Inf. téc. Pesca Chile, 47: 1-86.

Báez, P. y H. Andrade. 1977. Geryon affinis Milne Edwards y Bouvier 1894 frente a las costas de Chile (Crustacea, Decapoda, Brachyura, Geryonidae). An. Mus. Hist. Nat., Valparaíso, 10: 215-219.

Conan, G.Y. 1975. Pesca exploratoria con trampas en cerros submarinos del archipiélago de Juan Fernández. CIMAR, UCV, no publicado.

Dawson, E.W. y W.R. Webber. 1991. The deep-sea crab Chaceon ("Geryon"): a guide to information and a reference list of the family Geryonidae. Nat. Mus. N.Z. Misc. Ser., 24: 1-83.

Organización de Naciones Unidas para la Agricultura y la Alimentación (FAO). 1996. Estadísticas de pesca. FAO, Estadísticas de pesca, 82: 1-700.
Gneri, F.S. 1966. Conceptos sobre evaluación de efectivos pesqueros. CARPAS, T8: 1-30.

Gulland, J.A. (ed.). 1970. The fish resources of the ocean. FAO Fish. Tech. Paper, 97: 1-425.

Holt, S.J. 1956. Exploratory and experimental fishing. FAO Fish. Bull., 9(1): 1-22.

Krouse, J.S. 1989. Performance and selectivity of trap fisheries for crustaceans. En: J.F. Caddy (ed.). Marine invertebrate fisheries: their assessment and management. John Wiley and Sons, New York, pp. 307-325.

Retamal, M. y P. Arana. 2000. Descripción y distribución de cinco crustáceos decápodos recolectados en aguas profundas en torno a las islas Robinson Crusoe y Santa Clara (Archipiélago de Juan Fernández, Chile). Invest. Mar., Valparaíso, 28: $149-163$.

Troadec, J.-P. 1978. Fishing and assessment of stocks. FAO, Fish. Circ., 701: 1-7.

Williams, G.R. 1964. Organization and management of research on marine fish resources. FAO Fish. Tech. Paper, 43: 1-12. 